Eric G. Bend, PhD

Yue $\mathrm{Si}, \mathrm{PhD}$

David A. Stevenson, MD

Pinar Bayrak-Toydemir,

$\mathrm{MD}, \mathrm{PhD}$

Tara M. Newcomb, MS

Erik M. Jorgensen, PhD

Kathryn J. Swoboda, MD

Correspondence to

Dr. Swoboda:

kswoboda@mgh.harvard.edu

or Dr. Jorgensen:

jorgensen@biology.utah.edu

Supplemental data at Neurology.org

\section{NALCN channelopathies}

\section{Distinguishing gain-of-function and loss-of-function mutations OPEN}

\section{ABSTRACT}

Objective: To perform genotype-phenotype analysis in an infant with congenital arthrogryposis due to a de novo missense mutation in the NALCN ion channel and explore the mechanism of pathogenicity using a Caenorhabditis elegans model.

Methods: We performed whole-exome sequencing in a preterm neonate with congenital arthrogryposis and a severe life-threatening clinical course. We examined the mechanism of pathogenicity of the associated NALCN mutation by engineering the orthologous mutation into the nematode C elegans using CRISPR-Cas9.

Results: We identified a de novo missense mutation in NALCN, c.1768C $>T$, in an infant with a severe neonatal lethal form of the recently characterized CLIFAHDD syndrome (congenital contractures of the limbs and face with hypotonia and developmental delay). We report novel phenotypic features including prolonged episodes of stimulus-sensitive sustained muscular contraction associated with life-threatening episodes of desaturation and autonomic instability, extending the severity of previously described phenotypes associated with mutations in NALCN. When engineered into the $C$ elegans ortholog, this mutation results in a severe gain-of-function phenotype, with hypercontraction and uncoordinated movement. We engineered 6 additional CLIFAHDD syndrome mutations into $C$ elegans and the mechanism of action could be divided into 2 categories: half phenocopied gain-of-function mutants and half phenocopied loss-offunction mutants.

Conclusions: The clinical phenotype of our patient and electrophysiologic studies show sustained muscular contraction in response to transient sensory stimuli. In $C$ elegans, this mutation causes neuronal hyperactivity via a gain-of-function NALCN ion channel. Testing human variants of NALCN in $C$ elegans demonstrates that CLIFAHDD can be caused by dominant loss- or gainof-function mutations in ion channel function. Neurology ${ }^{\circledR}$ 2016;87:1131-1139

\section{GLOSSARY}

CLIFAHDD = congenital contractures of the limbs and face with hypotonia and developmental delay; $\mathbf{C R I S P R}=$ clustered regularly interspaced short palindromic repeats; IHPRF = infantile hypotonia with psychomotor retardation and characteristic facies.

NALCN is a conserved cation channel related to voltage-gated sodium and calcium channels. The NALCN family of channels is expressed throughout the nervous system in all metazoans studied and conducts a persistent sodium leak current that contributes to tonic neuronal excitability. ${ }^{1,2}$

Null mutations in orthologs of $N A L C N$ in mice, flies, and worms cause generalized paralysis with disruption of periodic behaviors such as breathing, circadian rhythms, or rhythmic motor circuits. ${ }^{1-4}$ Loss of these behaviors is likely caused by hyperpolarized membrane potentials in the neurons of these mutants. ${ }^{1,5}$ Loss-of-function mutations in the human NALCN have been

From the Department of Biology and Howard Hughes Medical Institute (E.G.B., E.M.J.), and Department of Pathology (Y.S., P.B.-T.), University of Utah, Salt Lake City; ARUP Institute for Clinical and Experimental Pathology (Y.S., P.B.-T.), Salt Lake City, UT; Division of Medical Genetics (D.A.S.), Department of Pediatrics, Stanford University, CA; Department of Neurology (T.M.N.), Pediatric Motor Disorders Research Program, University of Utah School of Medicine, Salt Lake City; and Department of Neurology (K.J.S.), Massachusetts General Hospital, Boston.

Go to Neurology.org for full disclosures. Funding information and disclosures deemed relevant by the authors, if any, are provided at the end of the article. The Article Processing Charge was paid by the authors.

This is an open access article distributed under the terms of the Creative Commons Attribution-NonCommercial-NoDerivatives License 4.0 (CC BY-NC-ND), which permits downloading and sharing the work provided it is properly cited. The work cannot be changed in any way or used commercially. 
described, which cause an autosomal recessive condition resulting in infantile hypotonia with psychomotor retardation and characteristic facies (IHPRF [MIM \#615419]). ${ }^{6-8}$ However, a new class of autosomal dominant NALCN mutations was identified in humans with a novel phenotype characterized by congenital distal arthrogryposis and pursed facial expression suggesting a hypercontracted phenotype-designated congenital contractures of the limbs and face with hypotonia and developmental delay (CLIFAHDD syndrome [MIM \#616266]).9,10 Because there is a significant degree of phenotypic overlap in patients with IHPRF, these mutations were hypothesized to function as dominant negative proteins that cause a loss-of-function phenotype. However, overexpressing a mutant NALCN ortho$\log$ in Caenorhabditis elegans led one group to conclude that the dominant channelopathy is caused by gain-of-function NALCN. ${ }^{1}$

To determine whether CLIFAHDD syndrome is caused by gain or loss of NALCN function, we engineered the orthologous missense mutation from our patient and 6 other published individuals into the $C$ elegans genome by CRISPR-Cas9. We found that the underlying pathologic mechanism of our patient's mutation is a gain-of-function change in NALCN. However, the additional mutations modeled in $C$ elegans demonstrate that the condition can be caused by both gainof-function and loss-of-function changes in the ion channel.

METHODS Family. The family described includes the proband, a preterm neonate born at 31 and 4/7 weeks' gestation, and her unaffected, nonconsanguineous parents of European heritage.

Standard protocol approvals, registrations, and patient consents. We obtained written informed consent for the collection of blood samples for DNA extraction. Counseling was performed before clinical whole-exome sequencing for all participants. The institutional review board at the University of Utah approved this study (IRB 25651, K.J.S.).

Exome sequencing and variant analysis. We performed diagnostic whole-exome sequencing in a trio using DNA extracted from whole blood. Genomic DNA was extracted using a Gentra Puregene Blood Kit (QIAGEN, Valencia, CA). Exons were captured with an Agilent SureSelect kit (Agilent Technologies, Inc., Santa Clara, CA) and sequenced with $2 \times$ 100 base-pair paired ends on an Illumina HiSeq 2500 (Illumina, Inc., San Diego, CA). The sequences were aligned to $\mathrm{Hg} 19$ using the Burrows-Wheeler Aligner (0.5.11), and variants were called with Genome Analysis Toolkit (v.1.6). More than $92.7 \%$ of bases sequenced had a quality score greater than 10 and variants with a quality score $<10$ were removed to avoid false positives. We excluded variants with an allele frequency greater than $1 \%$ in dbSNP (Single Nucleotide Polymorphism Database), 1000 Genomes Project, and 6500 Exomes. Further filtering removed synonymous variants, deep intronic variants, and those in $5^{\prime}$ and $3^{\prime}$ untranslated regions. We confirmed family relationships with short tandem repeat markers. The $N A L C N$ variant was confirmed by Sanger sequencing.

C elegans strains and genetics. Strains were cultured and maintained using standard methods. ${ }^{12}$ All strains and plasmids used in this study are listed (table e-1 at Neurology.org). CRISPR-Cas9 repair templates were made by Gibson cloning ${ }^{13}$ into pBluescript including: 1- to 3-kb homology arms with the orthologous NALCN mutation in nca-1, a loxP-flanked unc$119(+)$ positive selection cassette (intron 10), and a silent mutation in the PAM recognition site. Guide RNA constructs were made by Golden-Gate oligo annealing. Plasmid DNA was injected into the germline of unc-119(ed3) animals including Cas9-pDD162 (30 $\mathrm{ng} / \mu \mathrm{L}),{ }^{14}$ repair template $(30 \mathrm{ng} / \mu \mathrm{L})$, guide RNA (30 $\mathrm{ng} / \mu \mathrm{L}$ ), and coinjection markers for negative selection of extrachromosomal arrays. CRISPR events were detected by unc-119 rescue, survival following peel-1 expression, and the absence of red fluorescence expression. The wild-type control was made by isolating a CRISPR event that included the unc-119(+) selection, but no mutation. The unc-119(+) selection marker was excised from the genomes of all strains by injection of CRE recombinase-pDD104 ${ }^{14}$ with pBluescript $(50 \mathrm{ng} / \mu \mathrm{L})$ and coinjection markers.

C elegans analysis. Sequence alignment and amino acid positions are based on the rescuing isoform, $n c a-1 d$ (wormbase release WS247). Images were captured on a Zeiss Axioskop compound microscope with a $20 \times$ air objective (Carl Zeiss Microscopy, LLC, Thornwood, NY). Tracks were generated by animals crawling for 3 minutes on fresh OP50 plates. We photographed the tracks on a dissecting scope and traced them in Adobe Photoshop (Adobe Systems Inc., San Jose, CA). Behavioral scoring was conducted blind on plates staged with 10 L4-larva 24 hours before the scoring. All strains included in this study were scored together with replicates randomly mixed. Aldicarb plates were prepared with $2 \mathrm{mM}$ aldicarb in agar. All strains were assayed together in a blinded experiment with a replicate from each strain included for 6 trials. For a trial, 10 animals were placed per plate and paralysis was assessed every 15 minutes. Paralysis was scored based on complete cessation of movement and lack of response to a nose tap.

RESULTS Clinical assessment indicates sustained neuronal activity resulting in muscular hypercontraction. Prenatal/ neonatal course. The proband is a female infant born at 31 and 4/7 weeks of gestation, delivered via C-section following detection of polyhydramnios and abnormal fetal heart tracings. Respiratory distress was present from birth. Intubation was attempted but unsuccessful because of small mouth, microretrognathia, and jaw contracture. She ultimately required intubation in the operating room with complete paralysis. She was hypertensive from birth.

Dysmorphic features. Distinctive craniofacial features and distally predominant limb contractures were evident at birth (figure 1). 

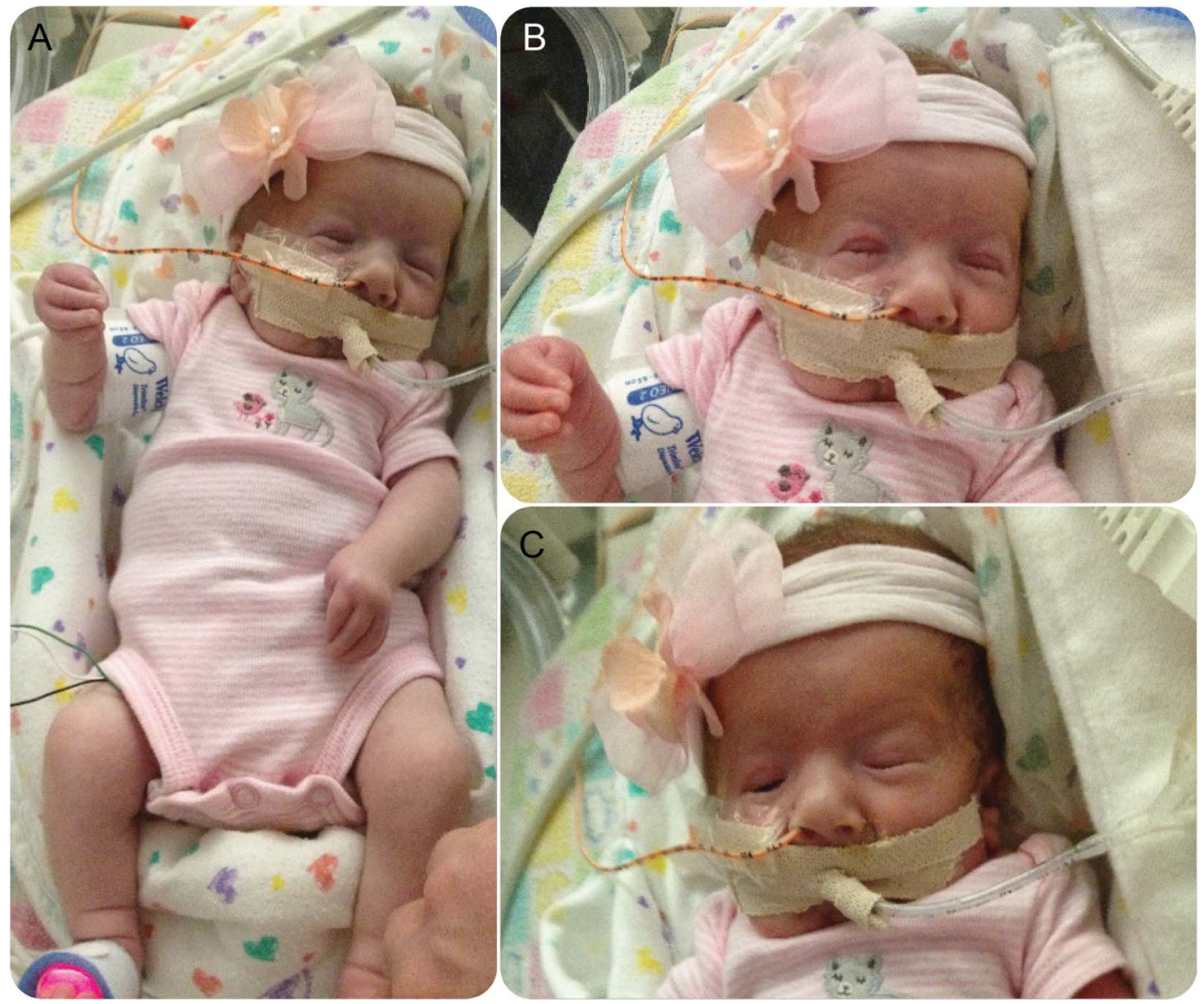

(A) Note overall characteristic pattern of limb contractures most significantly affecting distal upper extremities, with camptodactyly, adducted thumbs, and ulnar deviation, and less obvious foot, knee, and elbow contractures. (B) Notable facial features (partially obscured by endotracheal tube) include pursed lips and small mouth with associated jaw contracture, microretrognathia, eyelid and facial myotonia, short palpebral fissures, square face, and frontal bossing. (C) Facial appearance during period of relatively greater relaxation and attempted eye opening following partial resolution of eyelid myotonia.

Abnormal motor activity. She demonstrated rhythmic hypercontraction of arms and legs described as cycling, facial grimacing, and eyelid myotonia with inability to relax for up to several minutes. Such episodes occurred up to $80 \times$ per day, and treatment trials of carbamazepine, phenobarbital, levetiracetam, or phenytoin were ineffective; however, clonazepam modestly lessened the frequency and severity of rhythmic limb activity. With severe episodes, she developed whole body rigidity and oxygen desaturation despite ventilation.

Autonomic instability. Over the ensuing weeks, she experienced recurrent life-threatening autonomic crises associated with apnea, bradycardia, worsening hypertension, hyperthermia, and recurrent desaturation events in response to routine handling including suctioning or repositioning.

Electrophysiologic studies. Central nervous system. EEGs were performed on 4 occasions, followed by several days of continuous bedside monitoring for seizure activity. EEGs revealed abnormal background activity characterized by occasional rhythmic slowing. However, we observed no epileptiform activity during spells of rhythmic leg cycling, apnea, bradycardia, hypertension, or increased muscular tone.

Peripheral nervous system. EMG and nerve conduction testing demonstrated severely diminished ulnar, median, and peroneal compound muscle action potential amplitudes ( $<10 \%$ expected normal values) with preserved median sensory response. Needle insertion in both proximal and distal muscles during EMG elicited abnormal persistent motor unit recruitment for up to 5 minutes in association with sustained visible muscular contraction, despite the absence of further needle movement. Occasional neuromyotonia and complex repetitive discharges were observed, but were limited to distally innervated intrinsic hand muscles. No myotonia was observed in limb muscles, even after distal cooling. We noted moderately reduced recruitment of relatively normalappearing motor units in distally innervated muscles, but recruitment in more proximal muscles appeared 
normal. Overall, the pattern of abnormalities on EMG and nerve conduction studies supported a predominantly neurogenic process most severely affecting distally innervated limb muscles. However, the most striking clinical abnormality appeared to be a markedly delayed relaxation of muscles following activation resulting in involuntary sustained muscular contraction most predominantly affecting the masseter, perioral, and periocular facial muscles, laryngeal, chest, and proximal upper extremity muscles. One episode during EMG testing resulted in involuntary jaw closure and laryngeal muscular hyperactivity associated with desaturation persisting for a full 5 minutes. During this time, motor unit appearance and recruitment in the masseter muscle appeared entirely normal, but was involuntarily sustained, indicating possible motor neuron/axon hyperexcitability.

Neuroimaging studies. Brain MRI revealed punctate and linear nonhemorrhagic foci in the periventricular white matter on T2 fluid-attenuated inversion recovery and T1-weighted images, consistent with prematurity-associated injury. Magnetic resonance angiography and magnetic resonance spectroscopy were normal.

Clinical overview. Initial differential diagnoses included a variant of Freeman-Sheldon syndrome (FSS or DA2A $)^{15}$ based on the pattern of limb contractures and pursed facial appearance, or StuveWiedemann syndrome. ${ }^{16}$ Clinical genetic testing for mutations in $M Y H 3$ and LIFR were negative. At 2 months of age, tracheostomy and gastrostomy tubes were placed, but recurrent crises continued. Because of her increasing distress and inability to maintain sleep, comfort measures were instituted and respiratory support was withdrawn at 4.5 months of age. The patient died quickly following extubation and the parents declined further studies.

Exome sequencing identified a heterozygous mutation in $N A L C N$. Exome sequencing of the proband and parents identified a de novo mutation in NALCN (c.1768C >T; p.Leu590Phe) (figure e-1). The mutation was novel at the time, but the exact same mutation has been published since then in an unrelated patient with CLIFAHDD syndrome. ${ }^{9}$ The mutation maps to the S6 segment in the second domaina region that forms the channel gate in related voltage-gated sodium channels (figure 2, A and B). ${ }^{17,18}$ This mutation could create a constitutively open channel that results in a dominant gain-offunction defect. In addition, a duplication of approximately $409 \mathrm{~kb}$ at $4 \mathrm{q} 32.3$ was detected by SNP microarray. The genes in this region are not known to be associated with disease, and given the small size of the duplication, is unlikely to contribute to the patient's phenotype.
Validation of pathogenicity of NACLN mutation in $C$ elegans. To test this model, we turned to $C$ elegans, in which both loss-of-function and gain-of-function alleles have been characterized in the orthologous NCA channels. ${ }^{19}$ There are 2 redundant NALCN family members expressed in $C$ elegans, nca- 1 and nca-2. Loss-of-function mutations in both homologs causes a recessive phenotype: animals have normal body posture but when stimulated by touch, crawl away then suddenly halt in a stereotyped manner termed "fainting." By contrast, gain-of-function variants in nca-1 are dominant and result in hypertonia, smaller body size, and curly posture (figure 3B). ${ }^{19,20}$

We engineered the patient's NALCN mutation (L590F) into the orthologous position in the $C$ elegans gene using the CRISPR-Cas9 system to generate NCA-1(V637F) in the native locus (figure $3 \mathrm{~A}$ ). Animals with this single residue change resembled the characterized gain-of-function mutation NCA-1(A643V), which results in a constitutively open channel. ${ }^{19}$ Moreover, the strain with the human mutation was indistinguishable from the very severe gain-of-function mutant NCA-1(D647E). All 3 of these missense mutations led to small body size and curly posture compared to the wild-type control (figure 3B). We made the control strain by the same strategy as the mutant; however, no mutation was introduced. The mutants are easily distinguishable from the wild-type control in a blinded behavioral scoring assay (figure 3C). Given 3 minutes on fresh food, wild-type animals will explore a distance greater than $20 \mathrm{~mm}$. In contrast, animals with the $\mathrm{A} 643 \mathrm{~V}, \mathrm{D} 647 \mathrm{E}$, or V637F mutation display dramatically reduced locomotion (figure 3D). All 3 variants exhibit semidominant inheritance; heterozygous animals exhibit an intermediate phenotype (figures 3, B and D).

Uncoordinated locomotion and curly posture are consistent with neuronal dysfunction, but this phenotype could be attributable to an increase or decrease in synaptic transmission. To test neurotransmitter release, the animals were assayed for sensitivity to the acetylcholine esterase inhibitor aldicarb. This drug blocks acetylcholine degradation and causes hypercontraction and excitatory paralysis. ${ }^{21}$ Strains with excess transmission at neuromuscular junctions are hypersensitive to the paralyzing effect of the aldicarb. The known gain-of-function mutations (A643V and D647E) and the patient's mutation (V637F) caused aldicarb hypersensitivity, indicating an increase in neurotransmitter release at $C$ elegans neuromuscular junctions (figure $3 \mathrm{E}$ ). Of note, the null mutation in nca-1 has no effect on aldicarb sensitivity or behavior since the $n c a-1$ and $n c a-2$ genes are functionally 


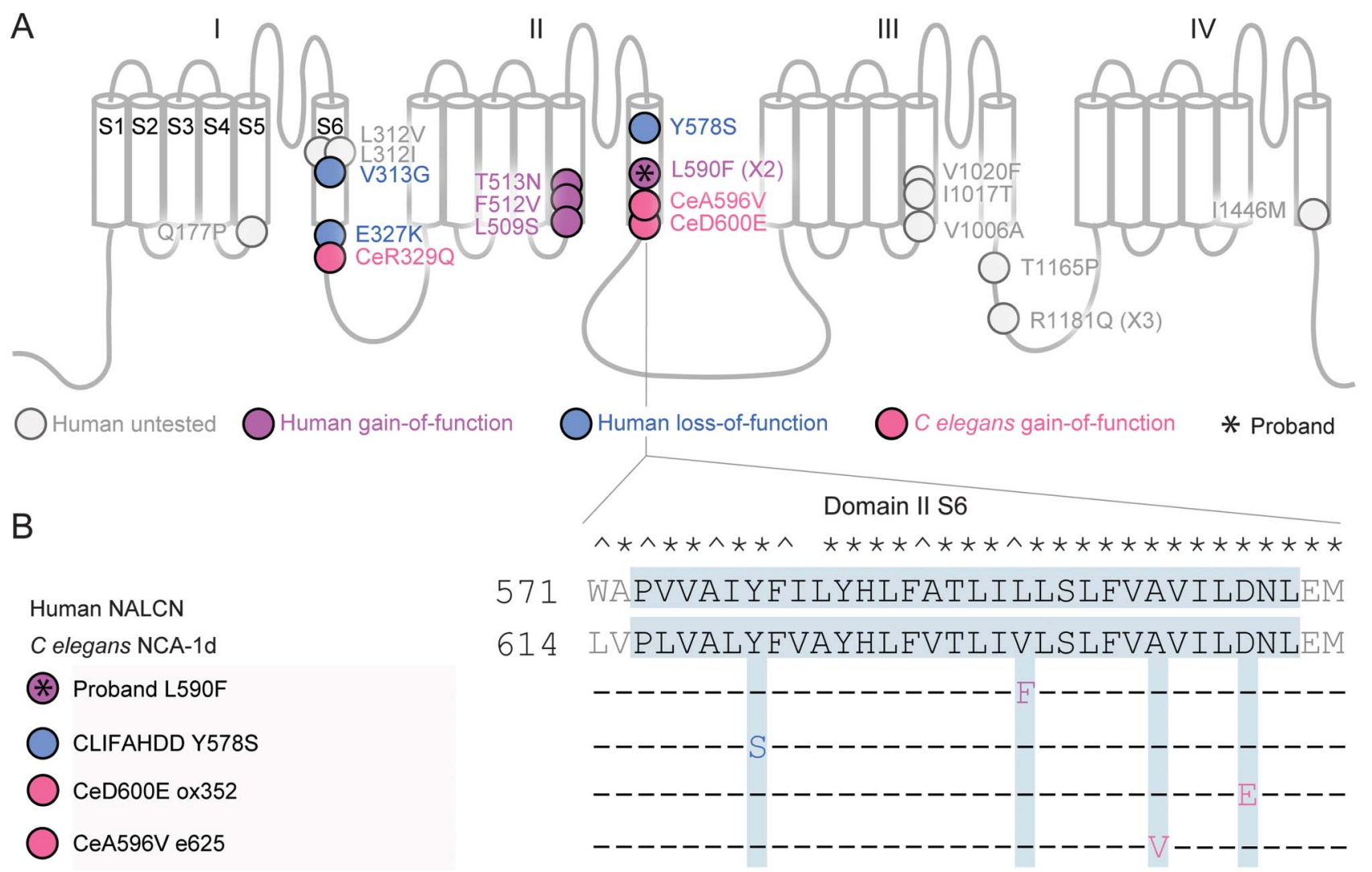

(A) Diagram of the NALCN predicted topology. Roman numerals label the domains and S1-6 indicate transmembrane segments. Missense mutations are displayed with circles and labeled with the human amino acid changes (for example, $C$ elegans ox352 D $\rightarrow E$ Ce 647/Hs 600). Multiple occurrences are indicated in parentheses (X\#). The proband's variant (*) maps near 2 known gain-of-function C elegans mutations (pink) in domain II S6. The remaining circles indicate all published human CLIFAHDD or worm gain-of-function mutations. ${ }^{8-11}$ Fill colors represent the functional results reported here (fuchsia $=$ gain-offunction; blue = loss-of-function; gray = not tested here). (B) The human and C elegans domain II S6 transmembrane segments (gray box) are aligned. Identical $(*)$ and similar $(\wedge)$ residues are indicated. Amino acid changes in human and nematode sequences are indicated below. CLIFAHDD $=$ congenital contractures of the limbs and face with hypotonia and developmental delay.

redundant. The D647E and V637E mutations are especially severe, and the time on aldicarb required to paralyze $50 \%$ of the worms (t1/2 for paralysis) is significantly shorter than the wild-type control (figure 3F). The A643V strain displayed a relatively weak hypersensitivity; however, the rate of paralysis (slope) was significantly greater than the wild-type control (figure 3G). Together, these findings support the classification of L590F in human NALCN as a dominant gain-of-function, pathogenic mutation.

Different CLIFAHDD mutations cause gain or loss of channel function in $\boldsymbol{C}$ elegans. To test whether all CLIFAHDD mutations cause a gain-of-function channel, we engineered 6 previously described missense changes into $C$ elegans nca-1 (figure 2A). ${ }^{9}$ We used the same CRISPR-Cas9 mutagenesis strategy. Including our patient's mutation, 7 of the 14 previously described CLIFAHDD mutations were tested. By scoring the cumulative behavior of the animals, we observed a spectrum of phenotypes ranging from wild-type to severely coiled (figure $4 \mathrm{~A})$. When these $n c a-1$ mutations were crossed into the nca-2(-) null background, 3 strains that appeared wild-type (V359G, E373K, Y625S) proved to be fainters (genotype nca-1[*/*] nca-2[-/-]). The fainting behavior was also observed when the mutations were heterozygous (genotype nca-1 $[* /+]$ nca-2[-/-]), indicating that they are antimorphs. Therefore, these mutations disrupt NCA-1 channel function in a dominant manner and should be classified as loss-of-function. The remaining 4 mutations displayed no fainting behavior in the nca2(-) null background; rather, the coiling behavior became subjectively more dramatic. The spectrum of postures observed in the strains with a noticeable phenotype is displayed (figure 4B). When assayed for aldicarb sensitivity, this subset of CLIFAHDD mutations caused a spectrum of hypersensitivity. The strains form an allelic series of severity based on the $t 1 / 2$ and the rate (slope) of paralysis, which resembles that determined by behavior 
A. CRISPR modification of nca-1

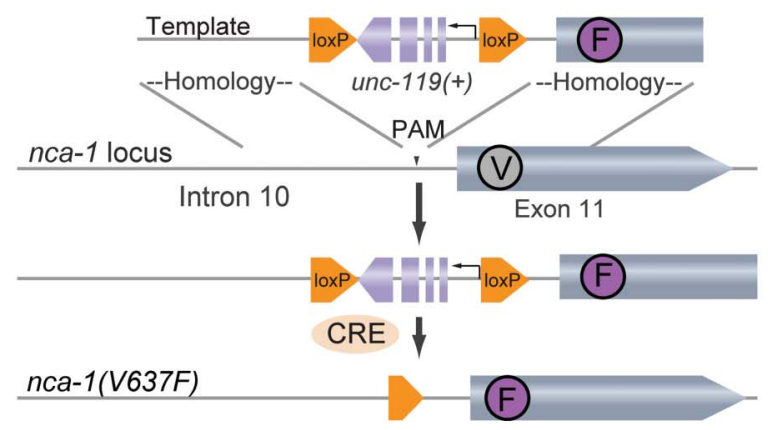

B. Posture of nca-1 variants

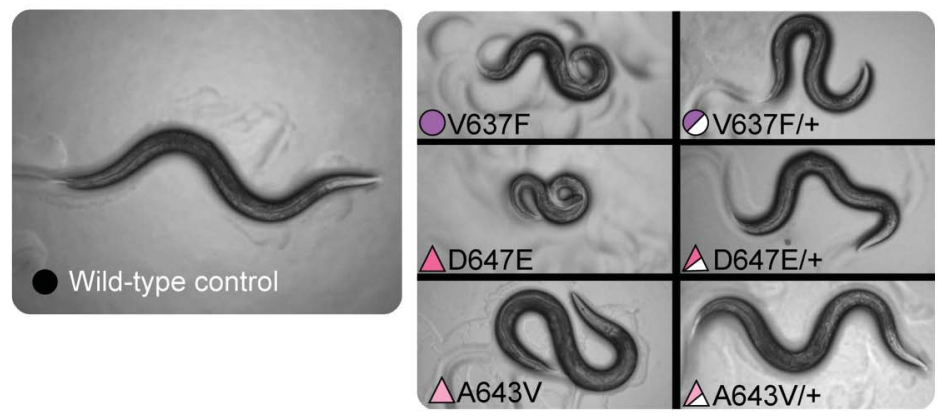

D. Locomotion of nca-1 variants

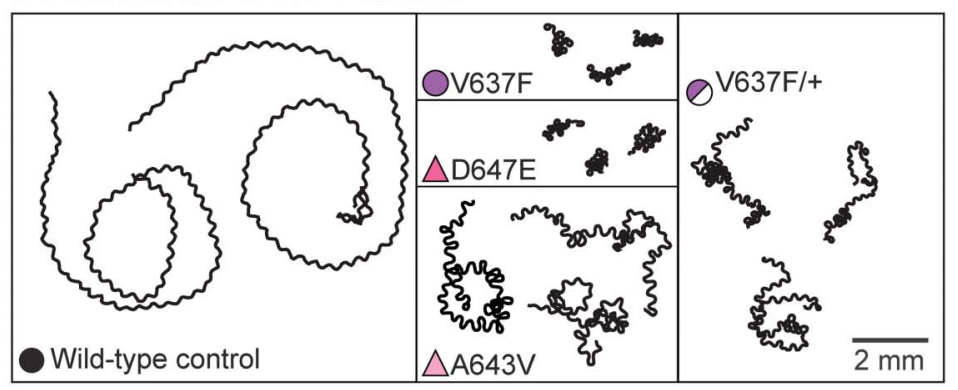

F. Aldicarb - Half paralyzed

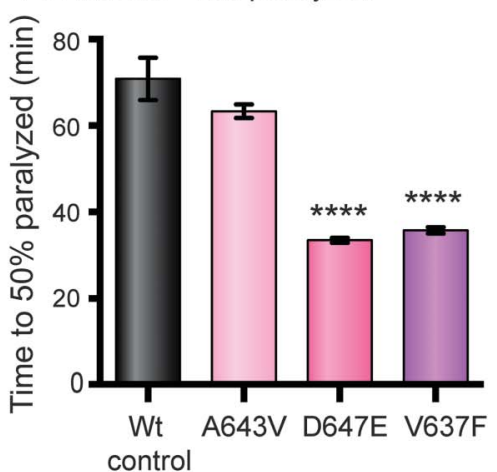

G. Aldicarb - Slope

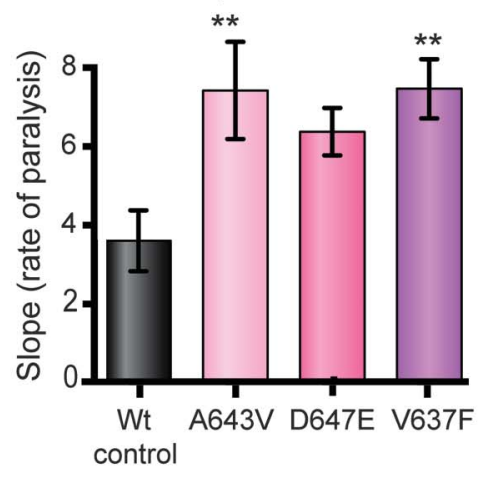

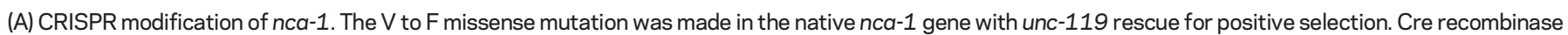

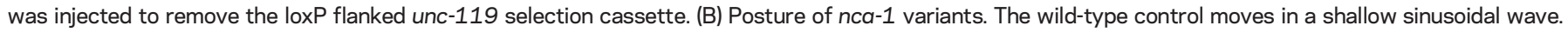

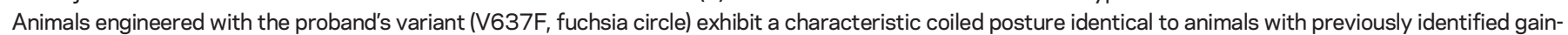

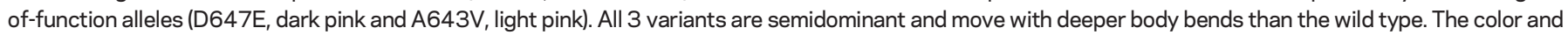

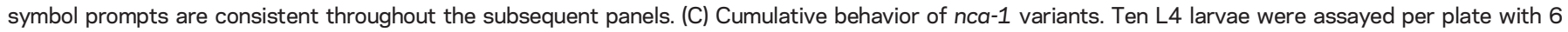

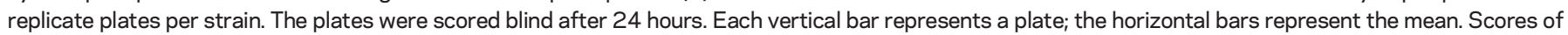

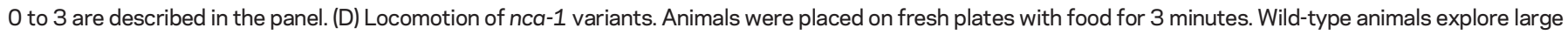

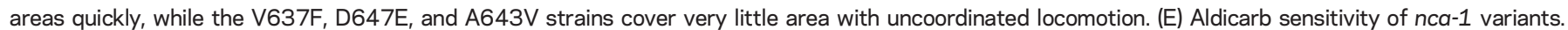

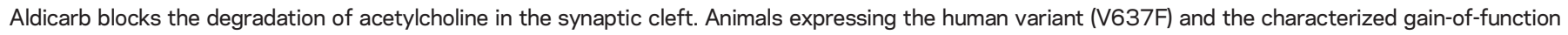

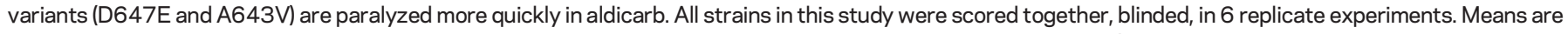

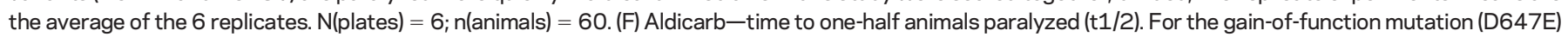

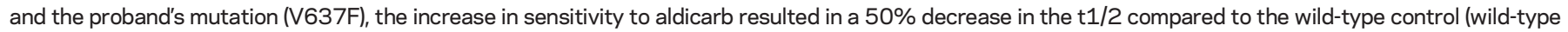

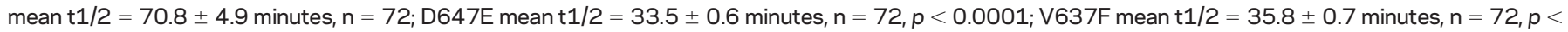

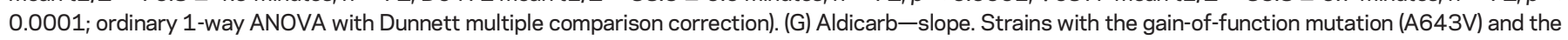

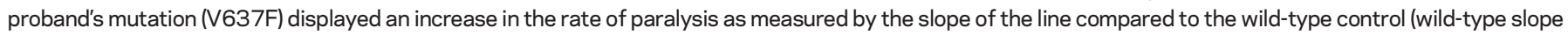

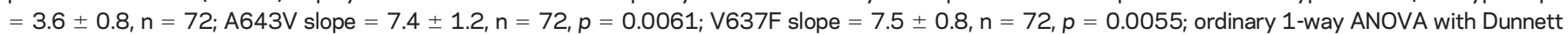
multiple comparison correction). Error bars are SEM; $* * p<0.01$, $* * * p<0.0001$. ANOVA $=$ analysis of variance; Wt $=$ wild-type.

(WT $<$ F559V $<$ T580N $<$ L556S $<$ V637F) (figure 4, $\mathrm{C}-\mathrm{E})$. In contrast, the mutations identified as fainters had no effect on aldicarb sensitivity (figure e-2). In summary, our results suggest that mutations responsible for CLIFAHDD syndrome may disrupt NALCN channel function by increasing or decreasing ion channel function.

DISCUSSION One problem emerging from diagnostic next-generation sequencing is the classification of 
A. Cumulative behavior of $C$ elegans strains with CLIFAHDD variants in nca-1
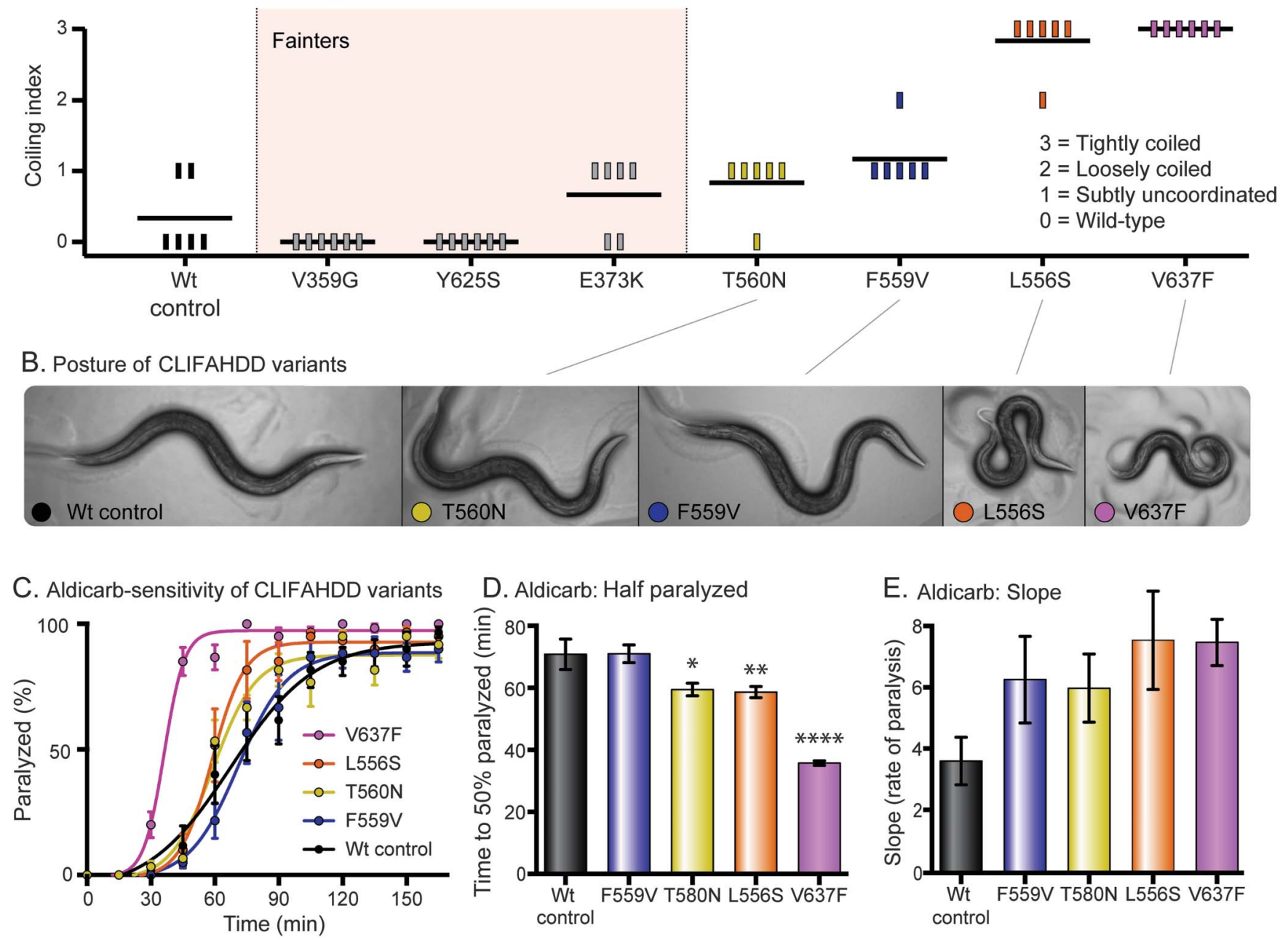

(A) Cumulative behavior of CLIFAHDD variants. Scores of 0 to 3 demonstrate the pathogenic severity of each mutation as indicated. When crossed into the nca-2(-) null background, the mutations highlighted in light pink displayed classic "fainting" behavior. (B) Posture of CLIFAHDD variants. The NCA-1 (T560N) and (F559V) strains have a subtle change in body posture with slightly deeper body bends than the wild type. The NCA-1(L556S) and (V637F) strains are strongly coiled. (C) Aldicarb sensitivity of CLIFAHDD variants. The uncoordinated/coiled subset of CLIFAHDD mutations causes aldicarb hypersensitivity with a spectrum of severity. The mutations determined to be loss-of-function by fainting behavior are not hypersensitive to aldicarb (figure e-2). (D) Aldicarb-time to one-half animals paralyzed (t1/2). The spectrum of aldicarb hypersensitivity is evident in the t1/2. The F559V mutant (t1/2 = 71.0 \pm 2.8 minutes, $n=72)$ was indistinguishable from the wild-type control (t1/2 = 70.8 \pm 4.9 minutes, $n=72)$. The T580N (t1/2 = 59.5 \pm 2.0 minutes, $n=72, p=0.0177)$, L556S (t1/2 = 58.6 \pm 1.8 minutes, $n=72, p=0.0095$ ), and V637F (t1/2 $=35.7 \pm 0.7$ minutes, $n=72, p<0.0001$ ) mutations displayed increasing sensitivity by this measure. (E) Aldicarb-slope. The rate of paralysis as captured by the slope displays a similar trend. However, the differences between the wild-type control and mutants do not reach significance (ordinary 1-way analysis of variance with Dunnett multiple comparison correction). Error bars are SEM; $* p<0.05, * * p<0.01,{ }^{* * * *} p<0.0001$. CLIFAHDD $=$ congenital contractures of the limbs and face with hypotonia and developmental delay.

novel genetic variants. This difficulty is compounded by phenotypic variation due to genetic background. A possible solution is to compare novel variants in a model organism with a fixed genetic background. Here, we used the nematode $C$ elegans to test 7 de novo mutations in the human NALCN gene responsible for CLIFAHDD syndrome. We found that all 7 variants were deleterious with dominantly inherited phenotypes. Thus, the worm appears to be a good model for CLIFAHDD_all mutations result in measurable phenotypes with the same inheritance pattern observed in humans.
Pathogenic mutations in NALCN can be categorized by different modes of inheritance. IHPRF syndrome is recessive and appears to be caused by null mutations since they truncate the NALCN protein. ${ }^{6-8}$ CLIFAHDD syndrome is dominantly inherited and caused by pathogenic missense mutations in NALCN.9 Previous reports disagree on the mechanism of CLIFAHDD suggesting it may result from protein loss- or gain-of-function. ${ }^{9-11}$ Our functional assays in $C$ elegans demonstrate that both outcomes are possible. We identified a subset of CLIFAHDD mutations with well-characterized gain-of-function 
phenotypes. These mutations cluster around the gate of the ion channel and likely increase current. By contrast, we also observed a class of mutations that act as dominant-negatives (antimorphs) in the nematode. Animals with these mutations display a stereotyped "fainting" behavior. Antimorphic channels likely cause the aggregation and degradation of wildtype NALCN, similar to mutations described in related sodium and calcium channels. ${ }^{22,23}$ Thus, 3 mechanisms give rise to NALCN channelopathies: (1) IHPRFrecessive loss-of-function, (2) CLIFAHDD—dominant gain-of-function, and (3) CLIFAHDD_dominant antimorphic.

Unfortunately, the human phenotypes that result from the different genetic mechanisms of NALCN pathology are not so easily placed into these categories. IHPRF and CLIFAHDD "syndromes" are characterized by dysmorphic features and neurodevelopmental disease with a significant number of

\section{Comment:}

\section{Genotype-phenotype correlation with CRISPR-Cas9-} Bedside to bench

Technological improvements and decreasing costs have led to increased use of next-generation sequencing as an a priori approach to clinical diagnosis. This approach lends itself to important discoveries of novel genotypic etiologies and phenotypic associations.

In the current report, the authors present a case of congenital arthrogryposis in an infant with a de novo missense mutation in the NALCN gene identified with whole-exome sequencing. ${ }^{1}$ Two groups originally reported NALCN mutations in 2013 in association with congenital contractures of the limbs and face with hypotonia and developmental delay (CLIFAHDD) syndrome. ${ }^{2,3}$ In contrast to earlier reports, Bend et al. describe in their patient the clinical electrophysiologic features of peripheral motor system hyperexcitability, thus expanding the phenotypic spectrum of NALCN-related disorders.

These findings are further investigated by probing the functional consequences of the orthologous missense NALCN mutation from their patient, as well as other previously reported NALCN mutations, using the CRISPR-Cas9 system in the model organism Caenorhabditis elegans. Consistent with their patient's clinical features of peripheral motor system overactivity, the authors nicely demonstrate in C elegans a gain of function as a consequence of the patient's mutation. Furthermore, other mutations previously reported in association with CLIFAHDD also had either loss- or gain-of-function consequences.

The authors' approach is an excellent example of how to use the CRISPRCas9 in a model system to investigate the functional consequences of missense mutations. The authors' use of motor behavior of $C$ elegans as a straightforward readout and the conserved nature of the NALCN gene makes the studies more easily interpreted. However, this paradigm may be less suited to the study of other disorders with more complex phenotypic-genotypic relationships, or in the study of less well conserved genes.

1. Bend EG, Si Y, Stevenson DA, et al. NALCN channelopathies: distinguishing gainof-function and loss-of-function mutations. Neurology 2016;87:1131-1139.

2. Köroğlu Ç, Seven M, Tolun A. Recessive truncating NALCN mutation in infantile neuroaxonal dystrophy with facial dysmorphism. J Med Genet 2013;50:515-520.

3. Al-Sayed MD, Al-Zaidan $\mathrm{H}$, Albakheet A, et al. Mutations in NALCN cause an autosomal-recessive syndrome with severe hypotonia, speech impairment, and cognitive delay. Am J Hum Genet 2013;93:721-726.

\section{W. David Arnold, MD}

From the Department of Neurology, Division of Neuromuscular Disorders, Department of PM\&R, and Department of Neuroscience, The Ohio State University Wexner Medical Center, Columbus, OH.

Study funding: No targeted funding reported.

Disclosure: W.D.A. is supported by a 3-year career development award from NIH/NICHD (5K12HD001097-17). Go to Neurology.org for full disclosures. shared features. Both IHPRF and CLIFAHDD patients display regional hypotonia and intellectual disability, and members of each group experience seizures. The most consistent feature attributed exclusively to CLIFAHDD syndrome is distal arthrogryposis (reported in 18 of 19 patients described to date) (figure 1). ${ }^{9-11}$ Distal contractures were not noted in the 11 patients described with IHPRF. ${ }^{6-8}$ Our results complicate this picture further, since it appears that gain- or loss-of-function mutations in $N A L C N$ can lead to a CLIFAHDD diagnosis. These results are consistent with the overlap between CLIFAHDD and IHPRF but at odds with the distinctive feature such as arthrogryposis. Given that NALCN is expressed in excitatory and inhibitory neurons, both sides of a balanced circuit will be affected by functional changes to the channel. Therefore, the motor output of a given circuit is difficult to predict and likely reflects homeostatic limits to the system. Therefore, the degree of phenotypic overlap between these syndromes may not be surprising.

The phenotypes observed in CLIFAHDD and IHPRF do suggest that the NALCN ion channel functions very broadly in the nervous system. Sustained muscular hyperactivity and failure of relaxation of limb and cranially innervated muscles, in conjunction with the observed rhythmic cycling activity of the limbs, suggest that this ion channel functions in both upper and lower motor neurons and associated circuitry in the peripheral nervous system as well as the CNS. Hyperexcitability of the motor unit and the resulting overactivity of muscles innervating both limbs and cranial structures, if present during fetal development, help to explain the characteristic dysmorphology and distally predominant congenital contractures observed in these patients.

Redefining the mechanisms of CLIFAHDD syndrome changes the approach for treating these patients. Gain-of-function variants could be targeted with ion channel blockers to decrease cellular excitability. Further functional studies are needed to identify specific blockers of NALCN. However, current medications in use for epilepsy or other indications may prove to be valuable candidates. Alternatively, NALCN variants identified with a loss-of-function mechanism may benefit from a global increase in cellular excitability. Unfortunately, both avenues will require extensive investigation and clinical trials. What is critical is that care providers practice caution before delivering such drugs because different patients with CLIFAHDD may respond in dramatically different ways. In particular, testing specific variants in $C$ elegans may lead to more accurate diagnoses and drug treatments. 


\section{AUTHOR CONTRIBUTIONS}

Eric G. Bend: design and conceptualization of the animal model studies, acquisition of data, analysis or interpretation of data, drafting/revising manuscript for content. Yue Si: acquisition of data, analysis or interpretation of data, drafting/revising manuscript for content. David A. Stevenson: acquisition of data, analysis or interpretation of data, drafting/ revising manuscript for content. Pinar Bayrak-Toydemir: acquisition of data, analysis or interpretation of data, drafting/revising manuscript for content. Tara M. Newcomb: drafting/revising the manuscript for content, study coordination. Erik M. Jorgensen: design and conceptualization of the animal model studies, analysis or interpretation of data, drafting/revising manuscript for content. Kathryn J. Swoboda: design and conceptualization of clinical investigation, data acquisition, drafting/revising the manuscript for content, analysis or interpretation of data, obtaining funding.

\section{ACKNOWLEDGMENT}

The authors thank the family for allowing their story and photos of their daughter to be shared. They also thank Rob Hobson for scoring fainting phenotypes and Pin-An Chen for the gift of NCA-1(D647E).

\section{STUDY FUNDING}

The National Center for Research Resources award ULRR025764 to the University of Utah Clinical Center for Translational Science provided core resources to help support this work, including the DNA extraction and sequencing core facilities.

\section{DISCLOSURE}

E. Bend, Y. Si, D. Stevenson, P. Bayrak-Toydemir, and T. Newcomb report no disclosures relevant to the manuscript. E. Jorgensen is a Howard Hughes Medical Institute Investigator. K. Swoboda is funded via research grants from the NIH (NICHD) (R01-HD69045 [NICHD]) and the CDC (U01-DD001108). Go to Neurology.org for full disclosures.

Received January 21, 2016. Accepted in final form May 4, 2016.

\section{REFERENCES}

1. Lu B, Su Y, Das S, Liu J, Xia J, Ren D. The neuronal channel NALCN contributes resting sodium permeability and is required for normal respiratory rhythm. Cell 2007; 129:371-383.

2. Gao S, Xie L, Kawano T, Po MD, Guan S, Zhen M. The NCA sodium leak channel is required for persistent motor circuit activity that sustains locomotion. Nat Commun 2015;6:6323.

3. Lear BC, Lin JM, Keath JR, McGill JJ, Raman IM, Allada R. The ion channel narrow abdomen is critical for neural output of the Drosophila circadian pacemaker. Neuron 2005;48:965-976.

4. Flourakis M, Kula-Eversole E, Hutchison AL, et al. A conserved bicycle model for circadian clock control of membrane excitability. Cell 2015;162:836-848.

5. Lu TZ, Feng ZP. A sodium leak current regulates pacemaker activity of adult central pattern generator neurons in Lymnaea stagnalis. PLoS One 2011;6:e18745.

6. Al-Sayed MD, Al-Zaidan H, Albakheet A, et al. Mutations in NALCN cause an autosomal-recessive syndrome with severe hypotonia, speech impairment, and cognitive delay. Am J Hum Genet 2013;93:721-726.

7. Köroğlu Ç, Seven M, Tolun A. Recessive truncating NALCN mutation in infantile neuroaxonal dystrophy with facial dysmorphism. J Med Genet 2013;50:515-520.
8. Gal M, Megen D, Zahran Y, et al. A novel homozygous splice site mutation in NALCN identified in siblings with cachexia, strabismus, severe intellectual disability, epilepsy and abnormal respiratory rhythm. Eur J Med Genet 2016; 59:204-209.

9. Chong JX, McMillin MJ, Shively KM, et al. De novo mutations in NALCN cause a syndrome characterized by congenital contractures of the limbs and face, hypotonia, and developmental delay. Am J Hum Genet 2015;96:462-473.

10. Fukai R, Saitsu H, Okamoto N, et al. De novo missense mutations in NALCN cause developmental and intellectual impairment with hypotonia. J Hum Genet 2016;61: 451-455.

11. Aoyagi K, Rossignol E, Hamdan FF, et al. A gain-offunction mutation in NALCN in a child with intellectual disability, ataxia, and arthrogryposis. Hum Mutat 2015; 36:753-757.

12. Brenner $\mathrm{S}$. The genetics of Caenorhabditis elegans. Genetics 1974;77:71-94.

13. Gibson DG, Benders GA, Andrews-Pfannkoch C, et al. Complete chemical synthesis, assembly, and cloning of a Mycoplasma genitalium genome. Science 2008;319: $1215-1220$.

14. Dickinson DJ, Ward JD, Reiner DJ, Goldstein B. Engineering the Caenorhabditis elegans genome using cas9triggered homologous recombination. Nat Methods 2013;10:1028-1034.

15. Toydemir RM, Rutherford A, Whitby FG, Jorde LB, Carey JC, Bamshad MJ. Mutations in embryonic myosin heavy chain (MYH3) cause Freeman-Sheldon syndrome and Sheldon-Hall syndrome. Nat Genet 2006; 38:561-565.

16. Dagoneau N, Scheffer D, Huber C, et al. Null leukemia inhibitory factor receptor (LIFR) mutations in Stüve-Wiedemann/Schwartz-Jampel type 2 syndrome. Am J Hum Genet 2004;74:298-305.

17. Payandeh J, Scheuer T, Zheng N, Catterall WA. The crystal structure of a voltage-gated sodium channel. Nature 2011;475:353-358.

18. Oelstrom K, Goldschen-Ohm MP, Holmgren M, Chanda B. Evolutionarily conserved intracellular gate of voltage-dependent sodium channels. Nat Commun 2014; 5:3420.

19. Yeh E, Ng S, Zhang M, et al. A putative cation channel, NCA-1, and a novel protein, UNC-80, transmit neuronal activity in C. elegans. PLoS Biol 2008;6:e55.

20. Xie L, Gao S, Alcaire SM, et al. NLF-1 delivers a sodium leak channel to regulate neuronal excitability and modulate rhythmic locomotion. Neuron 2013;77:1069-1082.

21. Miller KG, Alfonso A, Nguyen M, Crowell JA, Johnson CD, Rand JB. A genetic selection for Caenorhabditis elegans synaptic transmission mutants. Proc Natl Acad Sci USA 1996;93:12593-12598.

22. Kazusaku K, Kaneda M, Sugawara T, et al. A nonsense mutation of the sodium channel gene $S C N 2 A$ in a patient with intractable epilepsy and mental decline. J Neurosci 2004;24:2690-2698.

23. Rajakulendran S, Kaski D, Hanna MG. Neuronal P/Q-type calcium channel dysfunction in inherited disorders of the CNS. Nat Rev Neurol 2012;8:86-96. 


\section{Neurology}

\section{NALCN channelopathies: Distinguishing gain-of-function and loss-of-function mutations}

Eric G. Bend, Yue Si, David A. Stevenson, et al.

Neurology 2016;87;1131-1139 Published Online before print August 24, 2016

DOI 10.1212/WNL.0000000000003095

This information is current as of August 24, 2016

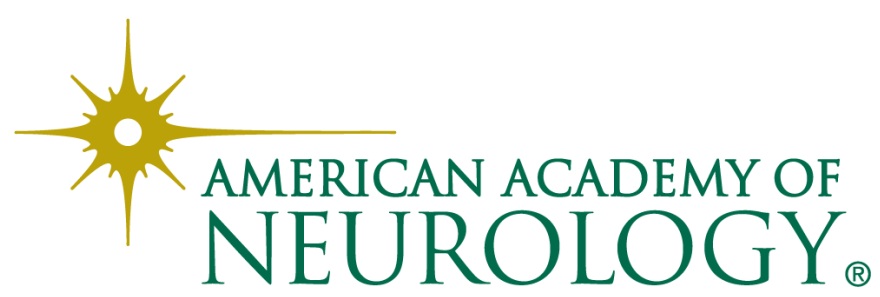




\section{Updated Information \& Services}

\section{Supplementary Material}

\section{References}

Citations

Subspecialty Collections

Permissions \& Licensing

\section{Reprints}

including high resolution figures, can be found at: http://n.neurology.org/content/87/11/1131.full

Supplementary material can be found at: http://n.neurology.org/content/suppl/2016/08/24/WNL.0000000000003 095.DC1

This article cites 23 articles, 5 of which you can access for free at: http://n.neurology.org/content/87/11/1131.full\#ref-list-1

This article has been cited by 1 HighWire-hosted articles: http://n.neurology.org/content/87/11/1131.full\#\#otherarticles

This article, along with others on similar topics, appears in the following collection(s):

\section{All Clinical Neurology}

http://n.neurology.org/cgi/collection/all_clinical_neurology

All clinical neurophysiology

http://n.neurology.org/cgi/collection/all_clinical_neurophysiology

Ion channel gene defects

http://n.neurology.org/cgi/collection/ion_channel_gene_defects

Neonatal

http://n.neurology.org/cgi/collection/neonatal

Neonatal seizures

http://n.neurology.org/cgi/collection/neonatal_seizures

Information about reproducing this article in parts (figures,tables) or in its entirety can be found online at:

http://www.neurology.org/about/about_the_journal\#permissions

Information about ordering reprints can be found online:

http://n.neurology.org/subscribers/advertise

Neurology ${ }^{\circledR}$ is the official journal of the American Academy of Neurology. Published continuously since 1951, it is now a weekly with 48 issues per year. Copyright @ 2016 American Academy of Neurology. All rights reserved. Print ISSN: 0028-3878. Online ISSN: 1526-632X.

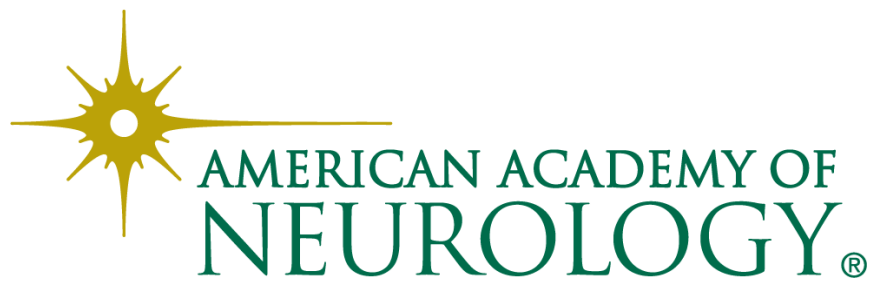

